Journal of Theoretical and Applied Mechanics, Sofia, Vol. 47 No. 4 (2017) pp. 75-85

\title{
NOVEL DYNAMIC MODEL UPDATING TECHNIQUE FOR DAMPED MECHANICAL SYSTEM
}

\author{
SHANKAR SEHGAL*, HARMESH KUMAR \\ Department of Mechanical Engineering UIET, Panjab University, \\ Chandigarh, 160 014, India
}

[Received 03 September 2016. Accepted 4 December 2017]

\begin{abstract}
Response surface method and Derringer's function approach have been combined together to develop novel structural dynamic model updating technique for a damped mechanical system. Response surface models have been incorporated instead of finite element models, in order to increase computational efficiency of proposed technique. Derringer's function approach is useful in dealing successfully with multi-objective optimization type model updating problems. Few such undamped techniques have been recently developed for undamped mechanical systems by combining the benefits of response models with Derringer's function approach. This paper presents the theory and numerical application of a damped updating technique, which is based upon response models and Derringer's function approach. In this technique, updating process is formulated as an optimization problem, wherein desirability functions are formulated, based on natural frequencies, modal-assurance-criterion values, resonance and anti-resonance points of frequency response functions. Desirability functions are then optimized to evaluate updated elastic parameters in stage one and updated damping constants in stage two of proposed technique. By using this technique, total absolute errors in natural frequencies, modal-assurance-criterion values, elastic parameters and frequency response functions have been reduced to $0.02 \%, 0.00 \%, 3.69 \%$ and $0.11 \%$, respectively.
\end{abstract}

KEY WORDS: Dynamic model updating; damped system; response surface method; derringer's function; analysis of variance.

\footnotetext{
${ }^{*}$ Corresponding author e-mail: sehgals@pu.ac.in
} 


\section{LIST OF SYMBOLS}

$[D] \quad$ damping matrix;

$[K] \quad$ stiffness matrix;

$[M] \quad$ mass matrix;

$D_{k} \quad$ coded parameter related to structural damping constant for stiffness matrix;

$D_{m} \quad$ coded parameter related to structural damping constant for mass matrix;

$\widehat{F R F}_{i} \quad$ response surface predicted $i^{\text {th }}$ peak / valley of frequency response function;

$\widehat{F R F}_{i L L} \quad$ lower limit for response surface predicted $i^{\text {th }}$ peak / valley of frequency response function;

$\widehat{F R F}_{i T} \quad$ target value for response surface predicted $i^{\text {th }}$ peak / valley of frequency response function;

$\widehat{F R F}_{i U L} \quad$ upper limit for response surface predicted $i^{\text {th }}$ peak / valley of frequency response function;

$\widehat{M A C}_{i i} \quad$ response surface predicted modal assurance criterion value for $i^{\text {th }}$ finite element mode with $i^{\text {th }}$ simulated experimental mode;

$\widehat{M A C}_{i i L L} \quad$ lower limit for response surface predicted modal assurance criterion value for $i^{\text {th }}$ finite element mode with $i^{\text {th }}$ simulated experimental mode;

$\widehat{M A C}_{i i U L} \quad$ upper limit for response surface predicted modal assurance criterion value for $i^{\text {th }}$ finite element mode with $i^{\text {th }}$ simulated experimental mode;

$d_{j} \quad j^{\text {th }}$ individual desirability function;

$i \quad$ integer;

$j \quad$ integer;

$n \quad$ total number of responses;

$\alpha \quad$ structural damping constant for mass matrix;

$\beta \quad$ structural damping constant for stiffness matrix;

$\widehat{\omega}_{i} \quad$ response surface predicted natural frequency of $i^{\text {th }}$ mode;

$\widehat{\omega}_{i L L} \quad$ lower limit for response surface predicted natural frequency of $i^{\text {th }}$ mode;

$\widehat{\omega}_{i T} \quad$ target value for response surface predicted natural frequency of $i^{\text {th }}$ mode;

$\widehat{\omega}_{i U L} \quad$ upper limit for response surface predicted natural frequency of $i^{\text {th }}$ mode.

\section{LIST OF ABBREVIATIONS}

ANOVA analysis of variance;

FE finite element;

FEMU finite element model updating;

FRF frequency response function;

IDF individual desirability function;
MAC modal assurance criterion;

ODF overall desirability function;

RS response surface;

RSM response surface method. 


\section{INTRODUCTION}

Due to advances in modal testing technology, it is a common practice to measure dynamic behaviour of products with good accuracy and precision. But Finite Element (FE) method is not able to predict the dynamic behaviour with complete accuracy, particularly if the structure is damaged. Hence, modal testing results are taken as targets and the system matrices of an FE model are updated by using Finite Element Model Updating (FEMU) techniques [1]. The early contributions, related to FEMU techniques are outlined in the survey paperson model updating in structural dynamics $[2,3]$.

Iterative FEMU techniques seem to be computationally inefficient, particularly, if applied to FE models of large size structures. Working on these directions, Sehgal and Kumar developed new iterative updating techniques, based upon combination of Response Surface Method (RSM) and Derringer's function approach [4-6]. In such techniques, objective functions were based upon natural frequencies and Modal Assurance Criterion (MAC) values, only. However, such techniques produce accurate results only for undamped systems. These techniques produce large errors in prediction of Frequency Response Functions (FRFs) of damped systems near resonance and anti-resonance points.

In this work, two-stage damped updating technique has been developed and successfully applied to solve a numerical updating problem. In the proposed technique; during first stage, mass and stiffness matrices are updated using the method of Sehgal and Kumar [5]. During second stage, updated mass and stiffness matrices are used in conjunction with resonance and anti-resonance points of FRFs in order to produce an updated damping matrix and hence, an updated damped FE model. Here, experimental FRFs and theoretical FRFs of undamped damaged beam have been compared and used to define the error indices based desirability functions, which were later optimized, using the proposed technique to evaluate the corrected damping constants of beam structure. Success of the technique is verified numerically by applying it for updating the FE model of a damaged damped cantilever beam structure.

\section{THEORETICAL DESCRIPTION OF PROPOSED UPDATING TECHNIQUE}

Proposed FEMU technique is based upon RSM and Derringer's function approach. Here, updating is performed in two stages, as explained in following sub-sections.

\subsection{First STAGE OF PROPOSED UPDATING TECHNIQUE}

Figure 1 shows the basic flow chart of first stage of proposed technique. During this stage, natural frequencies and MAC values of undamaged and damaged beams

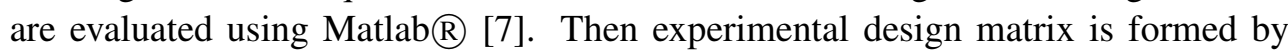




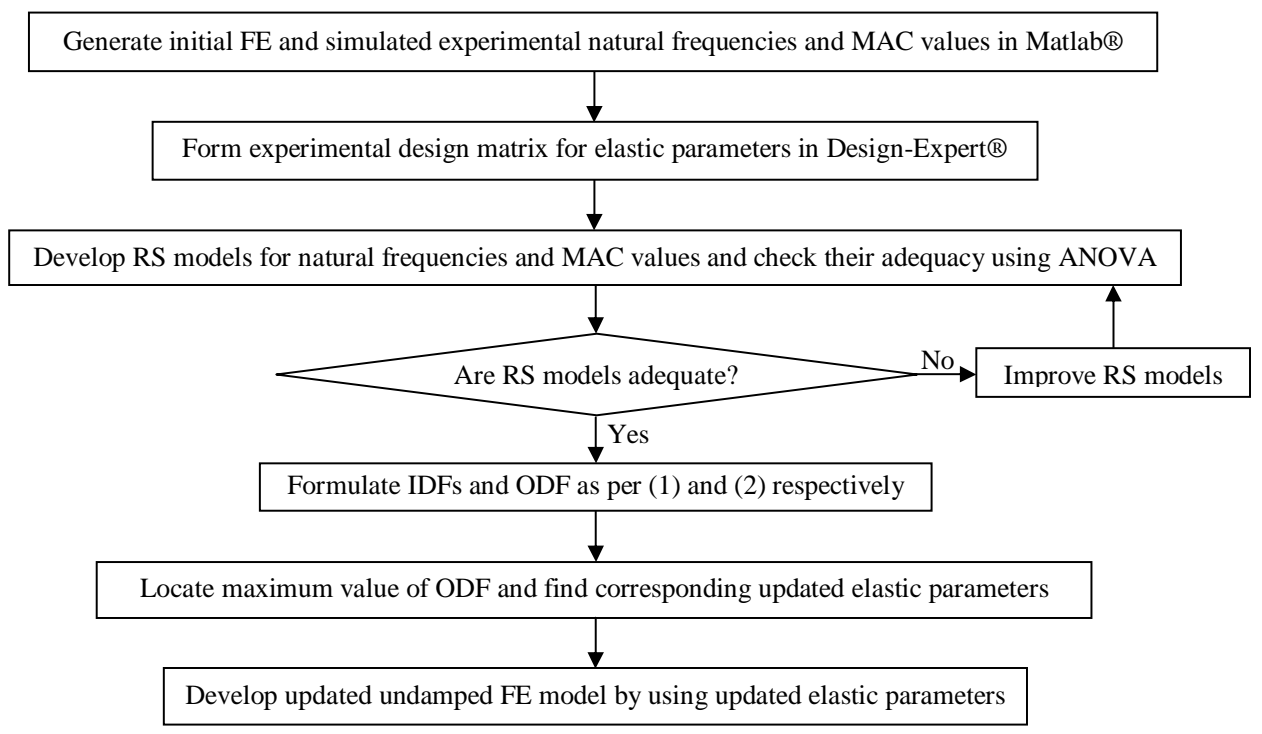

Fig. 1. Algorithm for first stage of updating.

using D-optimal design based values of elastic parameters in Design-Expert $($ software [8]. Experimental design matrix is then used to conduct simulated experi-

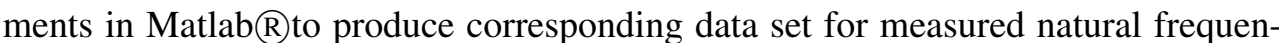
cies and MAC values. Regression analysis of this data set is performed to develop Response Surface (RS) models for natural frequencies and MAC values. RS models are then checked for accuracy and significance, by using ANalysis of VAriance (ANOVA) method. If response models are not good enough, then these are modified and checked again for their adequacy. After achieving adequate RS models for all response variables, Individual Desirability Functions (IDFs) are formulated using Derringer's function approach as per (1)

$$
\begin{aligned}
& \text { (1) }
\end{aligned}
$$

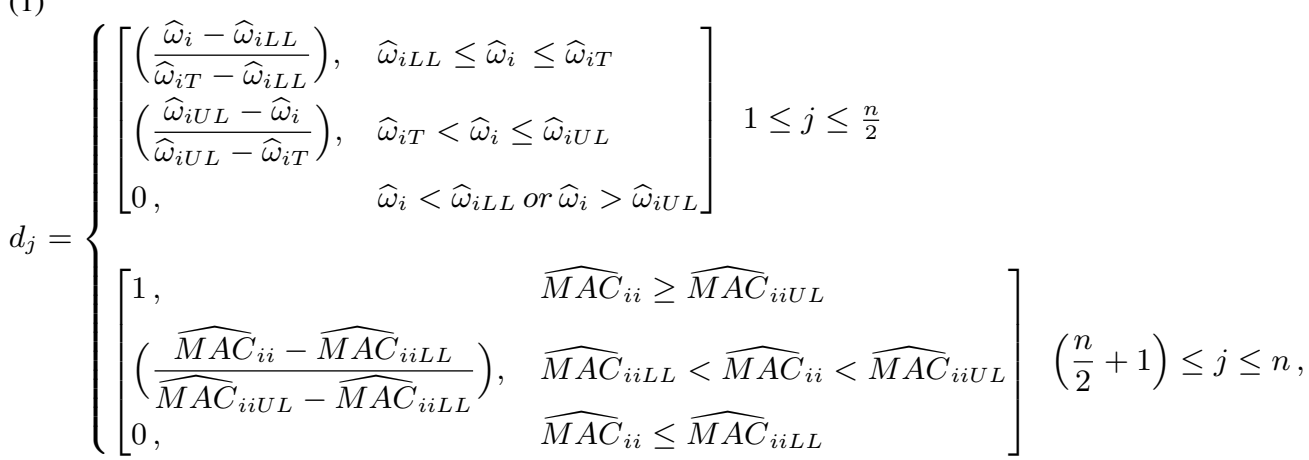


where $d_{j}$ is $j^{\text {th }}$ desirability function; $i$ and $j$ are integers; $n$ is the total number of responses; $\widehat{\omega}_{i}$ is response surface predicted natural frequency of $i^{\text {th }}$ mode; $\widehat{\omega}_{i L L}, \widehat{\omega}_{i T}$, $\widehat{\omega}_{i U L}$ are the lower limit, target value and upper limit of $\widehat{\omega}_{i}$, respectively; $\widehat{M A C}_{i i}$ is response surface predicted MAC value of $i^{\text {th }}$ finite element mode of undamaged beam with $i^{\text {th }}$ finite element mode of damaged beam; $\widehat{M A C}_{i i L L}$ and $\widehat{M A C}_{i i U L}$ are the lower limit and upper limit of $\widehat{M A C}_{i i}$, respectively.

All IDFs are then combined to produce an Overall Desirability Function (ODF) by using (2), thereby transforming the complex multi-objective type FEMU problem to a rather simpler single-objective type.

$$
\mathrm{ODF}=\left(d_{1} \times d_{2} \times d_{3} \times \ldots \times d_{n}\right)^{1 / n},
$$

where $n$ is total number of responses in the updating process.

RS model of ODF is developed. Maximum value of ODF is evaluated, using an algorithm available in the Design-Expert $\AA$ software. Thereafter, corresponding updated values of the elastic input parameters are extracted from the RS model of the ODF. These updated elastic parameters are then used to develop an updated un-

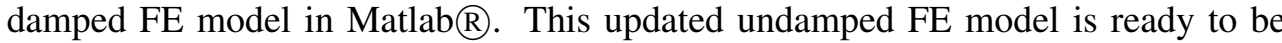
used as input in second stage of the technique.

\subsection{SECOND STAGE OF PROPOSED UPDATING TECHNIQUe}

As drawn in Fig. 2, during second stage, updated mass $[M]$ and stiffness $[K]$ matrices of first stage are taken as a starting point in conjunction with structural damping constants $\alpha$ and $\beta$ for finding out first approximation of damping matrix $[D]$ as per (3)

$$
[D]=\alpha[M]+\beta[K] .
$$

Second stage of updating aims at identifying the values of $\alpha$ and $\beta$, which are further processed to develop the updated damping matrix and updated damped dynamic responses, such as damped FRFs.

Updated mass and stiffness matrices of first stage of FEMU are used for developing FRFs of undamped damaged beam, which are then compared with the FRFs of damped damaged beam. Then, experimental design matrix is formed for structural damping constants $\alpha$ and $\beta$. This is performed by using D-optimal design method. Experimental design matrix is then used to produce corresponding data sets for peaks and valleys of FRFs. Then, regression analysis is used to develop RS models for peaks and valleys of FRFs. RS models are further checked for their accuracy and significance by using ANOVA method. If response models are not good enough then these are modified and checked again for their adequacy. After achieving adequate 


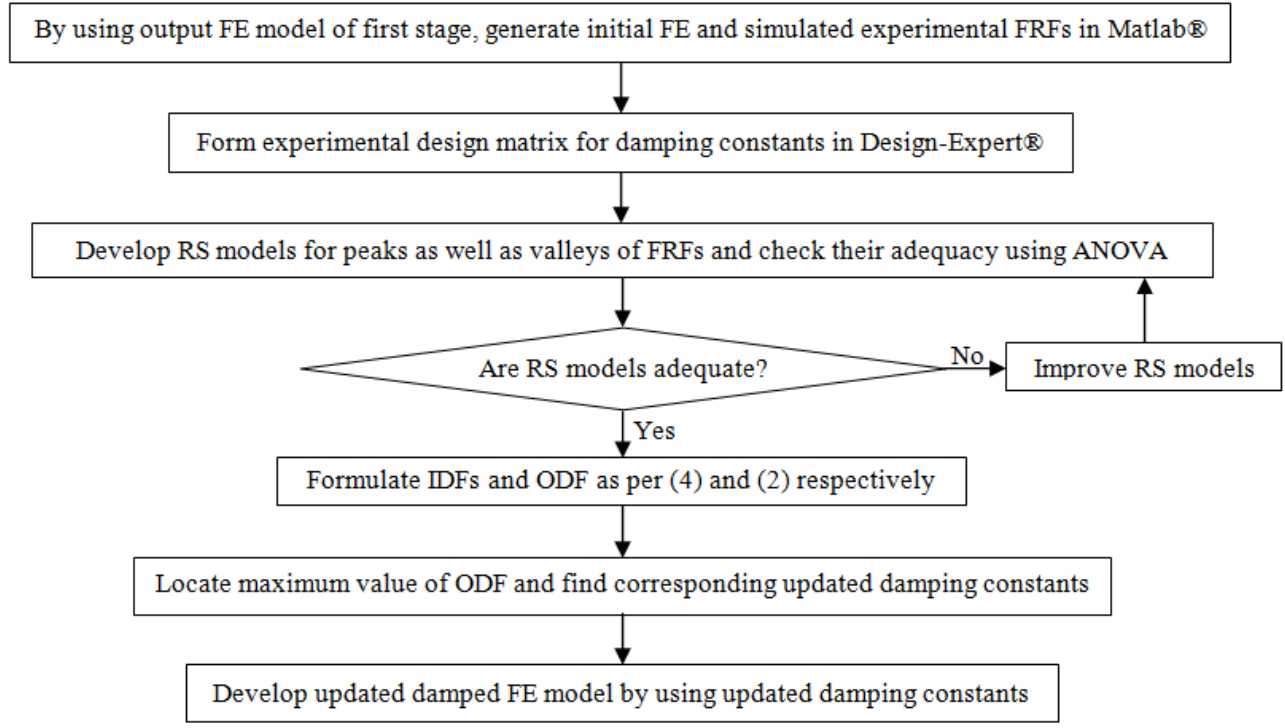

Fig. 2. Algorithm for second stage of updating.

RS models for all response variables, IDFs are formulated as per (4):

$$
d_{j}= \begin{cases}\widehat{F R F}_{i}-\widehat{F R F}_{i L L} & \widehat{F R F}_{i L L} \leq \widehat{F R F}_{i} \leq \widehat{F R F}_{i T}-\widehat{F R F}_{i L L}, \\ \widehat{F R F}_{i U L}-\widehat{F R F}_{i} & \widehat{F R F}_{i T}<\widehat{F R F}_{i} \leq \widehat{F R F}_{i U L} \\ 0, & \widehat{F R F}_{i}<\widehat{F R F}_{i L L} \text { or } \widehat{F R F}_{i}>\widehat{F R F}_{i U L}\end{cases}
$$

where, $d_{j}$ is the desirability function, related to $j^{\text {th }}$ peak / valley of frequency response function, $\widehat{F R F}_{i}$ is response surface predicted value of $i^{\text {th }}$ peak / valley of frequency response function, $\widehat{F R F}_{i L L}, \widehat{F R F}_{i T}, \widehat{F R F}_{i U L}$ are the lower limit, target value and upper limit of $\widehat{F R F}_{i}$, respectively.

All IDFs are then combined to produce an ODF by using (2) thereby transforming the complex multi-objective type FEMU problem to a rather simpler single-objective type. Maximum value of ODF is then searched out, which is further used to find out the updated values of structural damping constants of FE model. Updated values of damping constants are then used to produce the damping matrix and hence, an updated damped FE model, damped FRFs and corresponding error indices. Finally, an updated damped FE model and damped FRFs are obtained at the end of second stage of proposed updating technique. 


\section{NUMERICAL APPLICATION OF PROPOSED UPDATING TECHNIQUE}

Proposed FEMU technique has been used for updating the FE model of a damaged cantilever beam structure, shown in Fig. 3. The beam is having dimensions $910 \times$ $49 \times 7 \mathrm{~mm}^{3}$, density $6728 \mathrm{~kg} / \mathrm{m}^{3}$ and Young' modulus of elasticity as $200 \mathrm{GPa}$. Perturbation has been introduced into the FE model of damaged beam structure, by reducing the value of modulus of elasticity of six elements, as per the data provided in Table 1. Six damage locations have been selected in such a way, so as to distribute these over entire length of beam. Additionally, damping is added to the damaged beam by keeping both the structural damping constants, as 0.05 . Here, updating is performed in two stages. First stage of updating is used to evaluate the damages, while second stage is used to identify the updated damping matrix.

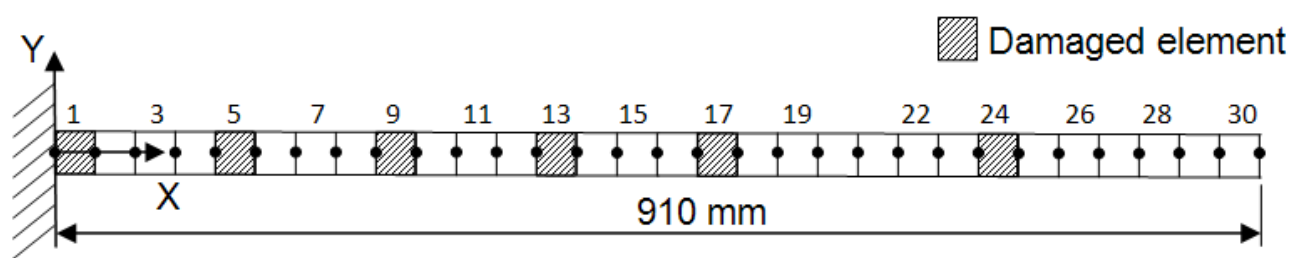

Fig. 3. FE model of a damaged cantilever beam.

Table 1. Elastic parameters of undamaged and damaged beams

\begin{tabular}{lcccccc}
\hline \hline Elastic parameter & $E_{1}$ & $E_{5}$ & $E_{9}$ & $E_{13}$ & $E_{17}$ & $E_{24}$ \\
\hline Stiffness in finite element model of undamaged beam $(\mathrm{GPa})$ & 200 & 200 & 200 & 200 & 200 & 200 \\
Stiffness in finite element model of damaged beam $(\mathrm{GPa})$ & 60 & 80 & 100 & 60 & 80 & 100 \\
\hline
\end{tabular}

\subsection{First STAGE DURING APPLICATION OF PROPOSED UPDATING TECHNIQUE}

FE model of undamaged, as well as damaged beams have been processed to produce corresponding natural frequencies and mode-shapes. Responses, such as natural frequencies of first five modes $\left(\omega_{1}\right.$ to $\left.\omega_{5}\right)$ and first five diagonal elements of MAC matrix $\left(M A C_{11}\right.$ to $\left.M A C_{55}\right)$ have been presented in Table 2.

It is clear from these results that responses of undamaged beam do not match with corresponding responses of damaged beam. So, input elastic parameters of FE model of undamaged beam were updated using first stage of the proposed FEMU technique. This was performed by using the objective functions, based upon refined response models of natural frequencies and MAC values [4]. Corresponding updated values of elastic constants for six damaged elements are: $60.21,80.37,99.59,59.25,80.74$ and $99.70 \mathrm{GPa}$. These updated values were used to produce an updated undamped FE 
Table 2. Natural frequencies and MAC values of undamaged and damaged beams

\begin{tabular}{|c|c|c|c|c|c|}
\hline \multirow[b]{2}{*}{$\begin{array}{l}\text { Response } \\
\text { variable }\end{array}$} & \multicolumn{2}{|c|}{ Response value (Hz) } & \multirow[b]{2}{*}{$\begin{array}{c}\text { Response } \\
\text { variable }\end{array}$} & \multicolumn{2}{|c|}{ Response value } \\
\hline & $\begin{array}{c}\text { Damaged } \\
\text { beam }\end{array}$ & $\begin{array}{c}\text { Undamaged } \\
\text { beam }\end{array}$ & & $\begin{array}{c}\text { Damaged } \\
\text { beam }\end{array}$ & $\begin{array}{c}\text { Undamaged } \\
\text { beam }\end{array}$ \\
\hline$\omega_{1}$ & 5.98 & 7.45 & $M A C_{11}$ & 1.0000 & 0.9996 \\
\hline$\omega_{2}$ & 37.91 & 46.66 & $M A C_{22}$ & 1.0000 & 0.9949 \\
\hline$\omega_{3}$ & 111.82 & 130.64 & $M A C_{33}$ & 1.0000 & 0.9873 \\
\hline$\omega_{4}$ & 213.02 & 256.01 & $M A C_{44}$ & 1.0000 & 0.9769 \\
\hline$\omega_{5}$ & 353.51 & 423.21 & $M A C_{55}$ & 1.0000 & 0.9544 \\
\hline
\end{tabular}

model, that showed an error reduction of $99.98 \%, 100.00 \%$ and $99.62 \%$ in prediction of natural frequencies, MAC values and elastic parameters, respectively. Thus, it was established that the first stage of proposed updating technique helps in almost completely removing the prediction errors, related to natural frequencies, MAC values and elastic parameters.

Figure 4 shows a comparison of FRFs of updated undamped beam, after first stage of updating with that of the damaged damped beam. Clearly, there is a big mismatch between the two FRFs near resonance and anti-resonance frequencies. The mismatch exists because of non-consideration of damping effects into the updated FE model produced by first stage of proposed technique. In order to remove the large errors, seen near peaks and valleys of FRFs, second stage of updating was performed as explained in next section.

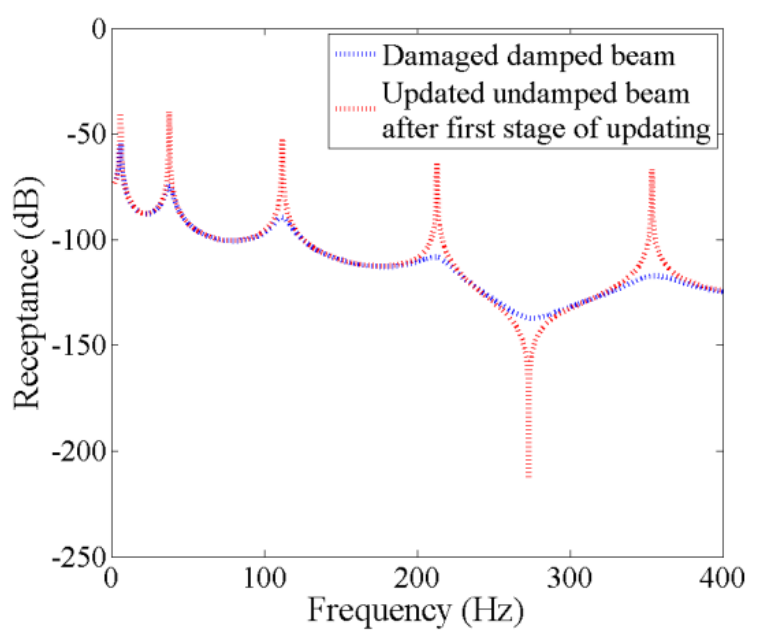

Fig. 4. Comparison of FRFs after first stage of updating. 


\subsection{SECOND STAGE DURING APPLICATION OF PROPOSED UPDATING TECH- NIQUE}

During second stage, the undamped updated results obtained during first stage of updating were taken as a starting point. Updated mass and stiffness matrices obtained in first stage were used in conjunction with the damping matrix obtained from (3) to generate RS models of peaks and valleys of FRF points. Variation of structural damping constants, $\alpha$ and $\beta$ were assumed to be within a range of 0.01 to 0.1 . Coded parameters $D_{m}$ and $D_{k}$ were defined in such a way that they varied linearly between -1 and +1 over the complete range of $\alpha$ and $\beta$ respectively. ANOVA was performed for all six responses (five peaks and one valley).

ANOVA results indicate that all the RS models were significant and having $\mathrm{p}$ value less than 0.0001 . The value of $\mathrm{R}^{2}$ and adjusted $\mathrm{R}^{2}$ was over $99 \%$, which is quite satisfactory. This means that the refined RS model gave very accurate relationship between input variables and response variables. Moreover, the "Predicted $\mathrm{R}^{2 \text { " value }}$ was also above $99 \%$ for all response variables, which was also in best agreement with "Adjusted $\mathrm{R}^{2}$ " value of above $99 \%$. Predicted residual error sum of squares was less than 0.2 for all response variables. Very low values of predicted residual error sum of squares show that polynomial models were fitting in an accurate manner. RS based polynomial equations for all responses have been given by the regression equations (5) to (10). IDFs were formulated by using (4) in conjunction with the numerical values provided in Table 3. IDFs were then processed further to form a single ODF as per (2).

$$
\begin{aligned}
\widehat{F R F}_{1}= & -55.19-2.92 \times 10^{-3} D_{m}-6.78 D_{k}+0.03 D_{m}^{2}+3.23 D_{k}^{2} \\
& -1.50 D_{k}^{3}, \\
\widehat{F R F}_{2}= & -75.86+1.63 \times 10^{-3} D_{m}-6.71 D_{k}+0.10 D_{m}^{2}+4.87 D_{k}^{2} \\
& -3.25 D_{k}^{3}, \\
\widehat{F R F}_{3}= & 90.38+1.75 \times 10^{-3} D_{m}-6.71 D_{k}+0.10 D_{m}^{2}+4.87 D_{k}^{2} \\
& -3.25 D_{k}^{3}, \\
\widehat{F R F}_{4}= & -109.18+1.78 \times 10^{-3} D_{m}-5.95 D_{k}+0.10 D_{m}^{2}+5.12 D_{k}^{2} \\
& -3.31 D_{k}^{3}, \\
\widehat{F R F}_{5}= & -136.39-1.79 \times 10^{-3} D_{m}+5.84 D_{k}-0.10 D_{m}^{2}-5.18 D_{k}^{2} \\
& +3.30 D_{k}^{3}, \\
\widehat{F R F}_{6}= & -118.18+1.79 \times 10^{-3} D_{m}-6.54 D_{k}+0.10 D_{m}^{2}+4.92 D_{k}^{2} \\
& -3.28 D_{k}^{3} .
\end{aligned}
$$


Table 3. Numerical details of IDFs, using RS models of FRFs

\begin{tabular}{cccc}
\hline $\begin{array}{c}\text { Response } \\
\text { surface predicted variable }\end{array}$ & $\begin{array}{c}\text { Target value } \\
(\mathrm{dB})\end{array}$ & $\begin{array}{c}\text { Lower limit } \\
(\mathrm{dB})\end{array}$ & $\begin{array}{c}\text { Upper limit } \\
(\mathrm{dB})\end{array}$ \\
\hline$\widehat{F R F}_{1}$ & -54.36 & -59.79 & -48.92 \\
$\widehat{F R F}_{2}$ & -74.87 & -82.35 & -67.38 \\
$\widehat{F R F}_{3}$ & -89.41 & -98.35 & -80.47 \\
$\widehat{F R F}_{4}$ & -108.29 & -119.12 & -97.46 \\
$\widehat{F R F}_{5}$ & -137.25 & -150.97 & -123.52 \\
$\widehat{F R F}_{6}$ & -117.18 & -128.90 & -105.46 \\
\hline
\end{tabular}

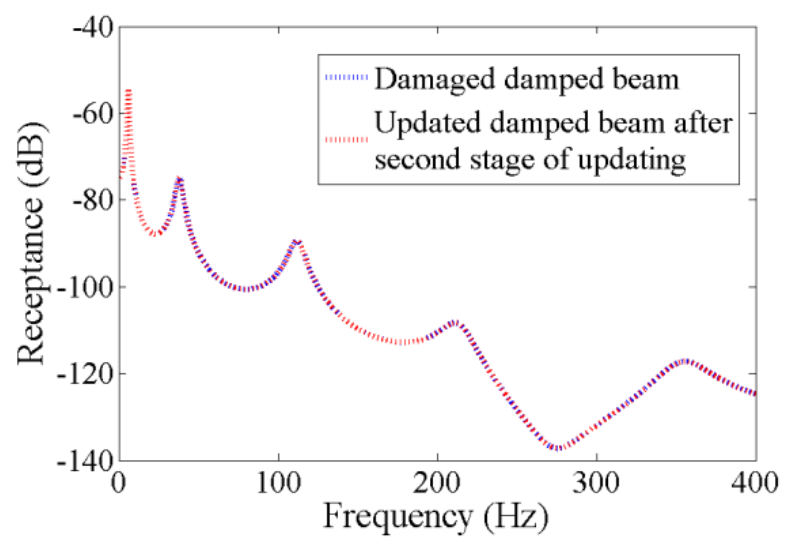

Fig. 5. Comparison of FRFs after second stage of updating.

Optimum value of the ODF was found to be 0.9981 . Corresponding set of updating parameters $(\alpha$ and $\beta$ ) was found to be (0.1 and 0.05), respectively. The updated values of the elastic parameters were then used to update the damping matrix and later on to produce updated damped FE model and damped FRFs. Updated damped FRFs have been then compared with the FRFs of damaged damped beam, as drawn in Fig. 5. It is seen that peaks and valleys of experimental and updated damped FE FRFs are now matching exactly. Before second stage, there was total absolute error of $253.02 \%$, considering all resonant and anti-resonant points, which has been reduced to just $0.11 \%$ after second stage of FEMU. With an error reduction of $99.96 \%$, the present FEMU technique has efficiently handled and reduced the errors in peaks and valleys of FE predicted FRFs. 


\section{CONCLUSION}

Damped updating technique has been designed and developed by combining the benefits of RSM with Derringer's function approach along with damping identification method. The technique has been successfully implemented for updating the model of a damped mechanical system. The technique has been verified numerically. Results prove that, percentage errors in prediction of natural frequencies, MAC values, elastic parameters and FRFs have been reduced by $99.98 \%, 100.00 \%, 99.62 \%$ and $99.6 \%$, respectively.

\section{ACKNOWLEDGMENTS}

Authors are grateful to University Grants Commission for financial support of this research work under major research project grant F. No. 42-869/2013(SR).

\section{REFERENCES}

[1] Friswell, M. I., J. E. Mottershead. Finite Element Model Updating in Structural Dynamics, The Netherlands: Kluwer Academic Publishers, 1995.

[2] Mottershead, J. E., M. I. Friswell. Model updating in Structural Dynamics: a Survey. Journal of Sound and Vibration, 167 (1993), No. 2, 347-375.

[3] Sehgal, S., H. Kumar. Structural Dynamic Model Updating Techniques: A State of The Art Review. Archives of Computational Methods in Engineering, 23 (2016), 515533.

[4] Sehgal, S., H. Kumar. Structural Dynamic Finite Element Model updating using Derringer's Function: a Novel Technique. WSEAS Transactions on Applied and Theoretical Mechanics, 9 (2014), 11-26.

[5] Sehgal, S., H. Kumar. Development of Benchmark Objective-function-formulation for Derringer's Function Based Model updating Method. WSEAS Transactions on Applied and Theoretical Mechanics, 9 (2014), 60-79.

[6] Sehgal, S., H. Kumar. Damage Detection using Derringer's Function bBsed Weighted Model updating Method, in Structural Health Monitoring, Volume 5: Proceedings of the $32^{\text {nd }}$ IMAC, A Conference and Exposition on Structural Dynamics, 2014, Conference Proceedings of the Society for Experimental Mechanics Series, A. Wicks, Ed. USA, Springer, 2014, 241-253.

[7] MATLAB $B$, User's guide of MATLAB v7, New York: The Mathworks Incorporation, 2004.

[8] Design-Expert $囚$, User's Guide for Version 8 of Design-Expert, Minneapolis, MN, StatEase Incorporation, 2010. 